\title{
Prevalence of 'Borderline' Values of Cardiovascular Risk Factors in the Clinical Practice of General Medicine in Italy Results of the BORDERLINE Study
}

Giuliano Tocci, ${ }_{1}^{1}$ Andrea Ferrucci, ${ }^{1}$ Jasmine Passerini, ${ }^{1}$ Maurizio Averna ${ }^{2}$ Paolo Bellotti ${ }^{3}$ Graziella Bruno, ${ }^{4}$ Francesco Cosentino, ${ }_{1}$ Gaetano Crepaldi, ${ }^{5}$ Cristina Giannattasio, ${ }^{6}$ Maria Grazia Modena, ${ }^{7}$ Giulio Nati, ${ }^{8}$ Antonio Tiengo, ${ }^{9}$ Bruno Trimarco, ${ }^{10}$ Diego Vanuzzo ${ }^{11}$ and Massimo Volpe, ${ }^{1,12}$ on behalf of the Italian Society for Cardiovascular Prevention (SIPREC)

1 Division of Cardiology, Department of Clinical and Molecular Medicine, Faculty of Medicine, University of Rome "Sapienza", Sant'Andrea Hospital, Rome, Italy

2 Department of Internal Medicine and Medical Specialties, University of Palermo, Palermo, Italy

3 Division of Cardiology, San Paolo Hospital, Savona, Italy

4 Department of Internal Medicine, University of Turin, Turin, Italy

5 CNR, Institute of Neuroscience, Section on Aging, Padova, Italy

6 Division of Clinical Medicine, Milano Bicocca University and San Gerardo Hospital, Monza, Italy

7 Division of Cardiology, Department of Emergency and Urgency, University of Modena, Modena, Italy

8 Italian Society of General Medicine (SIMG), Rome, Italy

9 Chair of Metabolic Disorders, Department of Clinical and Experimental Medicine, University of Padova, Padova, Italy

10 Department of Clinical Medicine, Cardiovascular and Immunological Sciences, Federico II University Hospital, Naples, Italy

11 Cardiovascular Prevention Centre, Health Unit 4 "Medio Friuli", Udine, Italy

12 IRCCS Neuromed, Pozzilli, Isernia, Italy

\section{Abstract}

Introduction: The prevalence of patients with 'borderline' levels of cardiovascular risk factors has been rarely investigated, being often reported in studies evaluating abnormal values of these parameters. The BORDERLINE study represents a pilot experience to primarily identify the prevalence of 'high-normal' conditions, such as pre-hypertension, lipid and glucose levels in the upper range of normality in the setting of general practice in Italy.

Aim: The aim of this study was to evaluate the prevalence of patients with 'borderline' values of cardiovascular risk factors in Italy.

Methods: Involved physicians were asked to evaluate the first 20 outpatients, consecutively seen in June 2009. Data were collected in a study-designed case-report form, in which physicians identified thresholds rather than reported absolute values of several clinical parameters. High-normal values were defined as follows: blood pressure (BP) 130-140/85-90 mmHg; total cholesterol 180-200 mg/dL; low-density lipoprotein cholesterol (LDL-C) 130-150 mg/dL; high-density lipoprotein cholesterol (HDL-C) 30-40 mg/dL in males and $40-50 \mathrm{mg} / \mathrm{dL}$ in females; triglycerides $130-150 \mathrm{mg} / \mathrm{dL}$ and fasting glucose $100-110 \mathrm{mg} / \mathrm{dL}$.

Results: Fifty-three Italian physicians provided valuable clinical data on 826 individual outpatients, among which $692\left(83.7 \%, 377\right.$ women, mean age $60.9 \pm 13.2$ years, body mass index $\left.26.6 \pm 5.0 \mathrm{~kg} / \mathrm{m}^{2}\right)$ were included in the present analysis. Prevalence of borderline values of systolic BP and total cholesterol levels were at least comparable with those in the normal limits of the corresponding parameters, whereas prevalence of borderline diastolic BP, LDL-C, HDL-C, triglycerides and fasting glucose levels was significantly lower than that of normal values, but higher than that of abnormal values of the corresponding parameters. 
Conclusions: Using this sample of healthy subjects in the setting of general practice in Italy, our results demonstrated a relatively high prevalence of borderline values of cardiovascular risk factors, which was at least comparable with that of normal, but significantly higher than that of abnormal thresholds. These preliminary findings may prompt more extensive investigations in the area of 'borderline' cardiovascular risk. This information may, in fact, potentially enable the design of more effective prevention strategies in the future to limit the burden of cardiovascular disease in the general population in Italy.

Received for publication 4 March 2011; accepted for publication 20 April 2011.

Keywords: borderline risk, high-normal risk, cardiovascular prevention, global cardiovascular risk, blood pressure, cholesterol, glucose.

\section{Introduction}

Cardiovascular disease still represents the leading cause of morbidity and mortality worldwide. ${ }^{[1]}$ Thus, strategies for cardiovascular disease prevention have progressively become a priority in the public agenda of national healthcare systems, mostly in Western countries. ${ }^{[2]}$ These strategies, however, have failed to substantially reduce cardiovascular morbidity and the overall burden of cardiovascular disease. ${ }^{[2]}$ This persistent gap between objective and attained results in terms of cardiovascular disease prevention may be due to several factors. Among these, the continuous increase of major cardiovascular risk factors, particularly arterial hypertension, hypercholesterolaemia, obesity, metabolic syndrome and diabetes mellitus, as well as the persistently high prevalence of unfavourable lifestyle habits (smoking, sedentary habits, high dietary intake) are largely responsible for the global burden of cardiovascular disease. ${ }^{[3]}$

During the last decade, international scientific societies have focused their attention on the clinical management of concomitant cardiovascular risk factors, proposing an integrated approach, in order to reduce the global cardiovascular risk of individual patients, rather than absolute levels of individual risk factors. ${ }^{[4]}$ Results from large observational studies have demonstrated the efficacy of such an approach only in those patients at high or very high cardiovascular risk profile. ${ }^{[5-8]}$ Modern medicine has, in fact, succeeded in significantly reducing mortality in the acute phase of cardiovascular disease and considerably ameliorated cardiovascular and renal outcomes. However, this strategy, based on intensive and integrated treatment of cardiovascular disease, cannot be applied to the general ('healthy') population, due to the high costs of diagnostic tools and pharmacological options. ${ }^{[9]}$

Recent findings seem to suggest the beneficial effects of early interventions on 'apparently healthy' (asymptomatic) individuals, who may be nonetheless at potential risk of experiencing major cardiovascular events, due to clustering of various cardiovascular risk factors or to the concomitant presence of subclinical organ damage. These interventions need to be applied before the risk of these subjects becomes elevated. For example, the TROPHY (TRial Of Preventing HYpertension) study ${ }^{[10]}$ has demonstrated the efficacy of treating subjects with pre-hypertension, whereas other studies have shown the importance of a therapeutic approach in patients with impaired fasting glucose and insulin resistance, ${ }^{[11,12]}$ and metabolic syndrome, ${ }^{[13-15]}$ in terms of reduced incidence of hypertension, diabetes, obesity and dyslipidaemia. Although preliminary, these results may be quite meaningful to design future preventive strategies to reduce the burden of cardiovascular disease.

On the basis of these considerations, the BORDERLINE study was designed as a pilot experience to investigate the prevalence of values in the high-normal range (borderline) for different cardiovascular risk factors. Thus, the primary aim of this survey was to identify the prevalence of 'borderline' conditions, i.e. patients with pre-hypertension, overweight and early abnormalities in glucose and lipid profiles, in the setting of general practice in Italy. This article presents and discusses the main findings of this study.

\section{Methods}

\section{Methodology of the Study}

The BORDERLINE study represents a preliminary experience, designed to evaluate the prevalence of patients with 'borderline' values of cardiovascular risk factors among outpatients followed by physicians operating in the setting of general medicine in Italy.

The study conformed to the Declaration of Helsinki and its subsequent modifications, and was approved by the reference Ethical Committee of the participating physicians. The confidentiality of the data was carefully and strictly protected. Written consent to participate in the programme was obtained by all involved physicians and patients. Confidentiality on demographic and clinical data of individual patients was carefully preserved. 


\section{Physicians' Recruitment}

Physicians' recruitment started in June 2009. Participants involved in the present study were randomly selected, in order to have a representative sample of the physicians in Italy, from a community of medical doctors who shared the following specific features: (i) experience in data collection and clinical case report form (CRF) compilation; and (ii) a routine practice of at least 60 patients per week, on average. Physicians involved in this pilot study were distributed in three main areas throughout the Italian territory (North, Centre and South).

Physicians were invited to participate in a pilot observational study, aimed at evaluating prevalence of borderline cardiovascular risk factors in their outpatients' population. Acceptance of this initial invitation placed physicians under no obligation, and physicians were entitled to drop out of the survey at any stage.

Written invitations were forwarded in a sizable number to ensure a sufficient representation of the study population sample, and to achieve this target within a period of approximately 3-4 weeks. Following their acceptance, involved physicians were asked to report clinical data, which were extracted from their clinical records from the first 20 consecutive adult Caucasian outpatients aged more than 30 years, whatever the reason they referred to their own attending physicians. The entire data collection was completed by participants on-site and the completed CRFs were then delivered to the Data Collection Centre by mail. At each study site, collection of data was conducted during 1 week within 1 month during summer 2009. Physicians who completed the programme did not receive any compensation for their participation.

The planned sample size of the survey participants included about 60 physicians in order to achieve an adequate representation of all Italian regions, as well as to limit excessive heterogeneity in age, gender, geographic location, professional expertise and practice size. The predefined minimum percentage of responses to achieve in order to declare the representative sample size was arbitrarily fixed to $80 \%$ of the total sample. Overall, the survey generated a population sample of 53 individual involved physicians ( $88.3 \%$ of the planned sample size) and reflected approximately an outpatient practice of about 1000 patients per week. Patients included in the present analysis were stratified in gender groups, according to the purpose of the study.

\section{Data Collection}

Data collection included full medical history and physical examination, anthropometric and clinical parameters, concomitant cardiovascular risk factors, current therapy for hy- pertension, lipid disorders, diabetes and other cardiovascular diseases, as well as any concomitant medications.

Based on anthropometric data, calculation was made of body mass index (BMI), which was expressed as bodyweight in kilograms divided by body surface in square metres. Clinic systolic and diastolic blood pressure (BP) levels, serum lipids, blood glucose and glycosylated haemoglobin $\left(\mathrm{HbA}_{1 \mathrm{c}}\right)$ levels, when available, were extracted from available clinical records and generally not exceeding 12 months.

Clinical data were collected in a specific CRF in which physicians identified thresholds rather than reported absolute values of major cardiovascular risk factors (see Supplemental Digital Content 1, http://links.adisonline.com/HBZ/A2). All parameters reported in the CRFs were derived from the clinical databases of enrolled physicians, if available, and were not measured at the time of the observation. Physicians were requested to report clinical data not exceeding 6 months before the observation.

\section{Data Analysis}

Available data were centrally analysed. Normal and abnormal values of different clinic and metabolic parameters were reported in a separate addendum and indicated as thresholds in the CRF. In particular, borderline values were considered as follows: BP $130-140 / 85-90 \mathrm{mmHg} ;{ }^{[16]}$ serum total cholesterol $180-200 \mathrm{mg} / \mathrm{dL} ;{ }^{[16]}$ low-density lipoprotein cholesterol (LDLC) $130-150 \mathrm{mg} / \mathrm{dL} ;{ }^{[16]}$ high-density lipoprotein cholesterol (HDL-C) $30-40 \mathrm{mg} / \mathrm{dL}$ in men and $40-50 \mathrm{mg} / \mathrm{dL}$ in women; ${ }^{[17,18]}$ triglyceride levels $130-150 \mathrm{mg} / \mathrm{dL} ; ;^{[17,18]}$ and fasting glucose $100-110 \mathrm{mg} / \mathrm{dL} \cdot{ }^{[19,20]}$

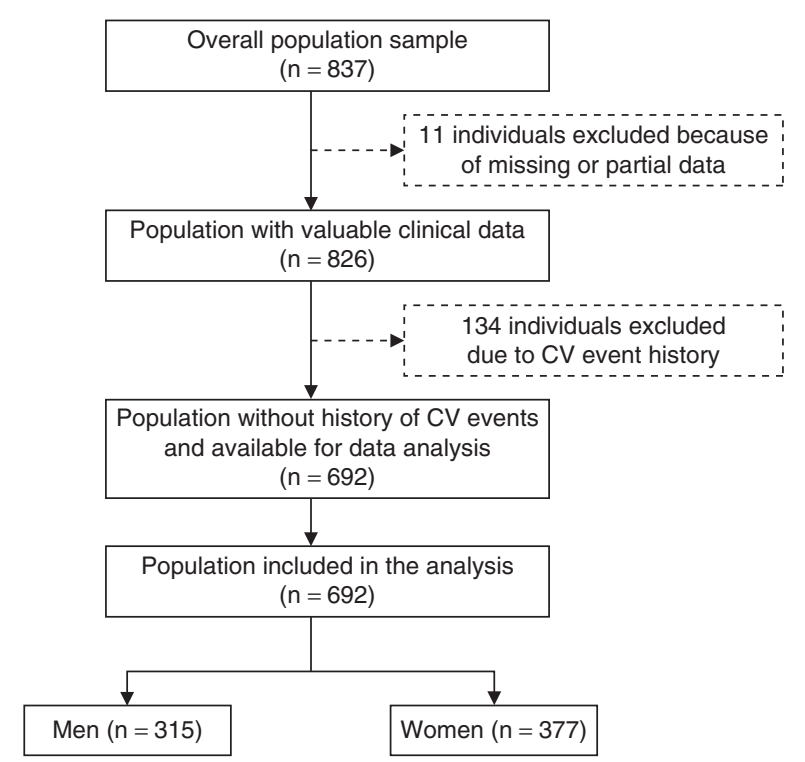

Fig. 1. Outpatients' selection for the analysis. $\mathbf{C V}=$ cardiovascular. 
Table I. General characteristics of the study population ${ }^{a}$

\begin{tabular}{|c|c|c|c|c|}
\hline Parameter & $\begin{array}{l}\text { Overall } \\
(n=692)\end{array}$ & $\begin{array}{l}\text { Men } \\
(n=315)\end{array}$ & $\begin{array}{l}\text { Women } \\
(n=377)\end{array}$ & $\begin{array}{l}\mathrm{p} \text {-Value } \\
\text { (Fisher) }\end{array}$ \\
\hline Age (y) & $60.9 \pm 13.2$ & $60.8 \pm 13.3$ & $61.0 \pm 13.2$ & \\
\hline Height (cm) & $169.9 \pm 8.4$ & $171.8 \pm 6.8$ & $161.2 \pm 6.3$ & $<0.05$ \\
\hline Weight $(\mathrm{kg})$ & $73.5 \pm 15.0$ & $79.7 \pm 14.0$ & $68.3 \pm 13.8$ & $<0.05$ \\
\hline Waist circumference $(\mathrm{cm})$ & $96.9 \pm 16.4$ & $100.0 \pm 17.4$ & $94.3 \pm 15.1$ & $<0.05$ \\
\hline Body mass index $\left(\mathrm{kg} / \mathrm{m}^{2}\right)$ & $26.6 \pm 5.0$ & $27.0 \pm 4.4$ & $26.3 \pm 5.4$ & \\
\hline Smoking (\%) & $169(24.4)$ & $97(30.8)$ & $72(19.1)$ & $<0.05$ \\
\hline Family history of hypertension (n) & $390(56.4)$ & $165(52.4)$ & $225(59.7)$ & \\
\hline Family history of diabetes mellitus ( $n$ ) & $208(30.1)$ & $97(30.8)$ & $111(29.4)$ & \\
\hline Hypertension (\%) & $411(59.4)$ & $203(67.6)$ & $208(55.1)$ & $<0.05$ \\
\hline Hypercholesterolaemia (\%) & $303(43.8)$ & $145(46.0)$ & $158(41.9)$ & \\
\hline Diabetes (\%) & $129(18.6)$ & $72(22.8)$ & $57(15.1)$ & $<0.05$ \\
\hline Obesity (\%) & $117(16.9)$ & $52(16.5)$ & $65(17.2)$ & $<0.05$ \\
\hline Creatininaemia $(\mathrm{mg} / \mathrm{dL})^{\mathrm{b}}$ & $1.0 \pm 0.5$ & $1.1 \pm 0.6$ & $0.9 \pm 0.5$ & \\
\hline Uric acid $(\mathrm{mg} / \mathrm{dL})^{\mathrm{b}}$ & $4.6 \pm 1.5$ & $5.9 \pm 1.6$ & $5.1 \pm 1.1$ & \\
\hline
\end{tabular}

a Data expressed as mean \pm SD. Numbers in parentheses are percentages by overall population.

b When available.

\section{Statistical Analysis}

All data were entered into Microsoft Access for Windows (Microsoft Office, Microsoft Corp., Redmond, WA, USA). Continuous variables were expressed as mean \pm standard deviation and compared by a chi-square test. Discrete variables were expressed as percentages and compared by Pearson's chisquared test. Because of the relatively small sample size, the comparisons were considered relevant for $\mathrm{p}<0.05$.

\section{Results}

\section{Population Sample}

As shown in figure 1, initially 53 Italian physicians reported information on 837 individual outpatients. Among these, 11 (1.3\%) records were omitted from the analysis because of missing or partial clinical data and 134 (16.2\%) outpatients were excluded because of a history of previous cardiovascular events (including coronary artery disease, stroke, peripheral artery disease or heart failure or diagnosed organ damage, renal disease or cancer). Thus, a total population sample of 692 otherwise healthy outpatients ( 377 women, mean age $60.9 \pm 13.2$ years, BMI $26.6 \pm 5.0 \mathrm{~kg} / \mathrm{m}^{2}$ ), which represents $83.7 \%$ of the original population sample, was included in the present analysis. General characteristics of the study population are reported in table I.

\section{Anthropometric and Clinical Parameters}

No significant difference was found regarding age and BMI between the two gender groups, although the men showed significantly higher values of height, weight and waist circumference than the women (table I). In the overall population sample, serum levels of both creatinine and uric acid were in the normal range, showing no significant differences between the two gender groups.

\section{Analysis of 'Borderline' Values}

The distribution of patients having normal, borderline and abnormal values of clinical parameters considered for the present analysis is reported in table II. The majority of individuals showed normal BP levels (i.e. $<130 / 85 \mathrm{mmHg}$ ), both for the systolic and for the diastolic BP levels (figure 2). Prevalence of borderline systolic BP levels was at least comparable with those within normal BP thresholds, while borderline diastolic BP levels were significantly less reported than those within normal BP thresholds $(\mathrm{p}<0.05)$. As expected, the simultaneous detection of systolic and diastolic BP levels in the high-normal range was relatively uncommon in this population sample of otherwise healthy subjects. Women showed a significantly higher prevalence of normal BP levels compared with men $(\mathrm{p}<0.05)$, whereas a significantly higher number of men showed border- 
line or abnormal BP values, both for the systolic and for the diastolic BP thresholds.

About $40 \%$ of the patients showed high levels of total cholesterol, while prevalence of values of total cholesterol in the upper-normal range was comparable with that of normal thresholds, and significantly lower than that of above-normal levels of this parameter (figure 3), without any significant difference between gender groups. In contrast, the majority of the patients showed normal values of LDL-C, followed by those having borderline and abnormal values of this parameter. In particular, borderline levels for LDL-C were reported in a significantly lower proportion of patients compared with normal levels and in a significantly higher proportion of patients having abnormal values of these parameters.

A significantly higher proportion of individuals of both genders had normal levels of both HDL-C and triglycerides compared with those who had borderline or abnormal levels of these parameters, as shown in figure 4. It should also be noted, however, that a significantly higher prevalence of borderline levels for HDL-C compared with that of above-normal levels

Table II. Absolute prevalence and percentage of normal, above-normal ('borderline') and abnormal thresholds for all clinical parameters considered in the analysis ${ }^{a}$

\begin{tabular}{|c|c|c|c|c|c|}
\hline Thresholds & $\begin{array}{l}\text { Overall } \\
(n=692)\end{array}$ & $\mathrm{p}$-Value (chi-square) & $\begin{array}{l}\text { Men } \\
(n=315)\end{array}$ & $\begin{array}{l}\text { Women } \\
(n=377)\end{array}$ & $\begin{array}{l}\mathrm{p} \text {-Value } \\
\text { (Fisher) }\end{array}$ \\
\hline \multicolumn{6}{|l|}{ SBP levels $(\mathrm{mmHg})$} \\
\hline$<130$ & $305(44.1)$ & & $124(39.4)$ & $181(48.0)$ & $<0.05$ \\
\hline $130-140$ & $278(40.2)$ & & $137(43.5)$ & $141(37.4)$ & $<0.05$ \\
\hline$>140$ & $91(13.2)$ & & $49(15.6)$ & $42(11.1)$ & $<0.05$ \\
\hline \multicolumn{6}{|l|}{ DBP levels $(\mathrm{mmHg})$} \\
\hline$<85$ & $422(61.0)$ & $<0.01$ & $170(54.0)$ & $252(66.8)$ & $<0.05$ \\
\hline $85-90$ & $206(29.8)$ & & $110(34.9)$ & $96(25.5)$ & $<0.05$ \\
\hline$>90$ & $45(6.5)$ & & $30(9.5)$ & $15(4.0)$ & $<0.05$ \\
\hline \multicolumn{6}{|c|}{ Total cholesterol levels (mg/dL) } \\
\hline$<180$ & $176(25.7)$ & $<0.01$ & $90(28.6)$ & $88(23.3)$ & \\
\hline $180-200$ & $193(27.9)$ & & $83(26.3)$ & $110(29.2)$ & \\
\hline$>200$ & $271(39.2)$ & & $122(38.7)$ & $149(39.5)$ & \\
\hline \multicolumn{6}{|l|}{ LDL-C levels (mg/dL) } \\
\hline$<130$ & $292(42.2)$ & $<0.01$ & $140(44.4)$ & $152(40.3)$ & \\
\hline $130-150$ & $162(23.4)$ & & $66(21.0)$ & $96(25.5)$ & $<0.05$ \\
\hline$>150$ & $117(16.9)$ & & $60(19.0)$ & $57(15.1)$ & $<0.05$ \\
\hline \multicolumn{6}{|l|}{ HDL-C levels (mg/dL) } \\
\hline Men >40; women >50 & $405(58.5)$ & $<0.01$ & $174(55.2)$ & $231(61.3)$ & $<0.05$ \\
\hline Men 30-40; women 40-50 & $200(28.9)$ & & $101(32.1)$ & $99(26.3)$ & $<0.05$ \\
\hline Men <30; women $<40$ & $20(2.9)$ & & $14(4.4)$ & $6(1.6)$ & $<0.05$ \\
\hline \multicolumn{6}{|c|}{ Triglycerides levels (mg/dL) } \\
\hline$<130$ & $306(44.2)$ & $<0.01$ & $131(41.6)$ & $175(46.4)$ & \\
\hline $130-150$ & $165(23.8)$ & & $74(23.5)$ & $91(24.1)$ & \\
\hline$>150$ & $146(21.1)$ & & $82(26.0)$ & $64(17.0)$ & $<0.05$ \\
\hline \multicolumn{6}{|c|}{ Fasting glucose levels (mg/dL) } \\
\hline$<100$ & $382(55.2)$ & $<0.01$ & $155(49.2)$ & $227(60.2)$ & $<0.05$ \\
\hline $100-110$ & $130(18.2)$ & & $70(22.2)$ & $60(15.9)$ & $<0.05$ \\
\hline$>110$ & 99 (14.3) & & $55(17.5)$ & $44(11.7)$ & $<0.05$ \\
\hline
\end{tabular}

a Numbers in parentheses are percentages by overall population.

DBP = diastolic blood pressure; HDL-C=high-density lipoprotein cholesterol; LDL-C =low-density lipoprotein cholesterol; $\mathbf{S B P}=$ systolic blood pressure 


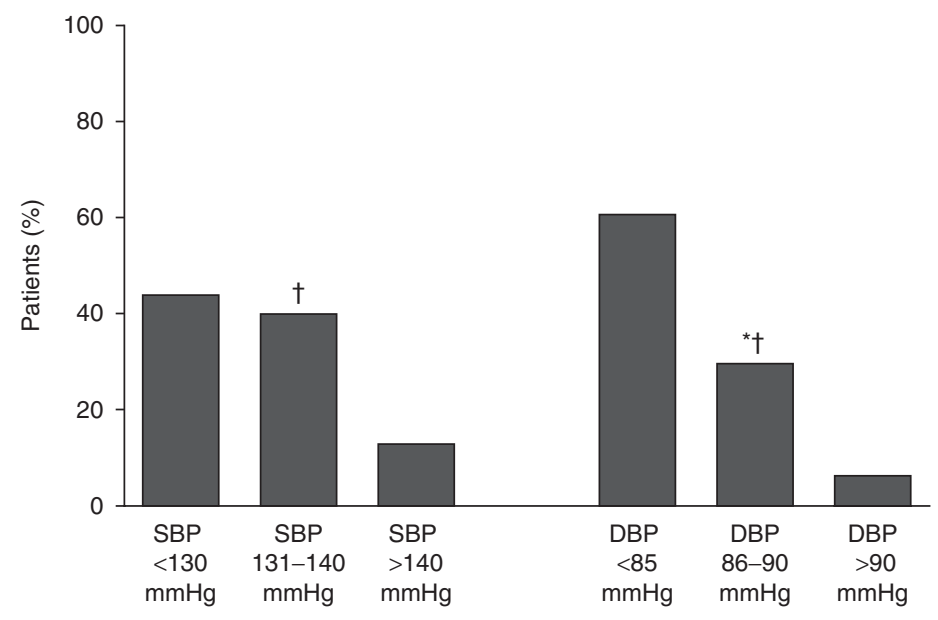

Fig. 2. Prevalence of normal, high-normal ('borderline') and abnormal thresholds for systolic (SBP) and diastolic (DBP) blood pressure levels. Data are expressed as percentages of the overall population sample. ${ }^{*} p<0.05$ vs normal thresholds; $\uparrow p<0.05$ vs abnormal thresholds.

was reported both for both men and women, whereas no significant difference was found between these two categories with respect to triglyceride levels.

Finally, a significantly higher proportion of individuals, mostly women, had normal fasting glucose levels, compared with that having borderline values of glucose profile. No significant difference was found between borderline and abnormal glucose thresholds, both in men and women, as shown in figure 5 . A similar prevalence of subjects having normal or high-normal BMI (overweight) was found, which was significantly higher than that of subjects with high values of BMI. In particular, a significantly higher proportion of men showed borderline values of BMI than that reported in women, who were significantly more prevalent in the normal thresholds of BMI.

\section{Discussion}

The present manuscript reports the analysis of a pilot observational study, performed in Italy, in the setting of daily clinical practice. The primary aim of this study was to collect information on the prevalence of high-normal ('borderline') levels of major cardiovascular factors in outpatients followed by general practitioners in Italy. The results of this analysis are somehow surprising and unexpected, since they demonstrated a relatively high prevalence of borderline values of cardiovascular risk factors, which was at least comparable with that of normal, but significantly higher than that of abnormal thresholds in a cohort of otherwise healthy subjects in Italy. Among the numerous data made available by this analysis, some specific aspects deserve discussion.
First, to our knowledge, this is the first experience primarily designed to evaluate the prevalence of borderline abnormalities of major cardiovascular and metabolic risk factors in a setting of general clinical practice. Similar data, in fact, have been provided by the Progetto Cuore ${ }^{[21]}$ of the Italian Ministry of Health, which analysed prevalence and absolute levels of major cardiovascular and metabolic risk factors in a much larger representative sample of the Italian general population, thus providing information of both borderline and above-normal values of the same clinical parameters and other clinical and population items. This analysis, however, was based on clinical data derived from individuals of both sexes, aged between 35 and 74 years, who were examined between 1998 and 2002. Our analysis, although limited to a smaller population sample than that included in the Progetto Cuore, ${ }^{[21]}$ can be considered more up to date and more focused on subjects with borderline abnormalities of different clinical parameters relevant to cardiovascular risk. In this latter regard, it should also be mentioned that the normal and abnormal thresholds applied in the Progetto Cuore ${ }^{[21]}$ are frankly higher than those used in our analysis, which strictly reflects the most recent guidelines for the diagnosis and treatment of hypertension, ${ }^{[16]} \operatorname{lipid}^{[17,18]}$ and glucose $^{[19,20]}$ abnormalities. For example, in the Progetto Cuore $^{[21]}$ arterial hypertension was defined as systolic and/or diastolic BP levels above 160/95 mmHg (borderline BP levels: 140-159/90-94 mmHg), while hypercholesterolaemia was defined as serum total cholesterol levels above $240 \mathrm{mg} / \mathrm{dL}$ (borderline total cholesterol: $200-239 \mathrm{mg} / \mathrm{dL}$ ). These thresholds have been substantially modified in the most recent guidelines. These differences in the diagnostic criteria may at least, in part,

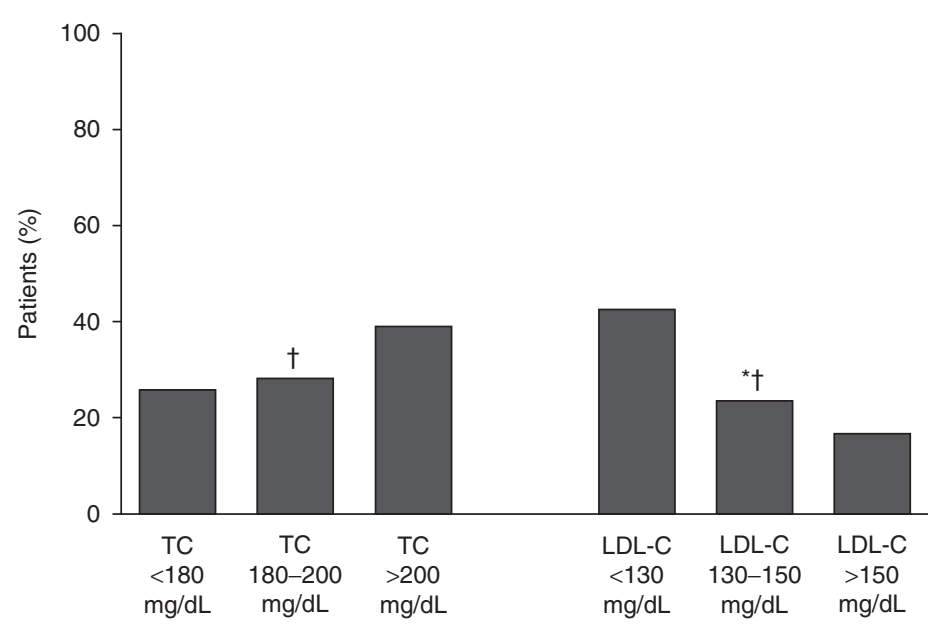

Fig. 3. Prevalence of normal, high-normal ('borderline') and abnormal thresholds for total cholesterol (TC) and low-density lipoprotein cholesterol (LDL-C) levels. Data are expressed as percentages of the overall population sample. ${ }^{*} p<0.05$ vs normal thresholds; $+p<0.05$ vs abnormal thresholds. 


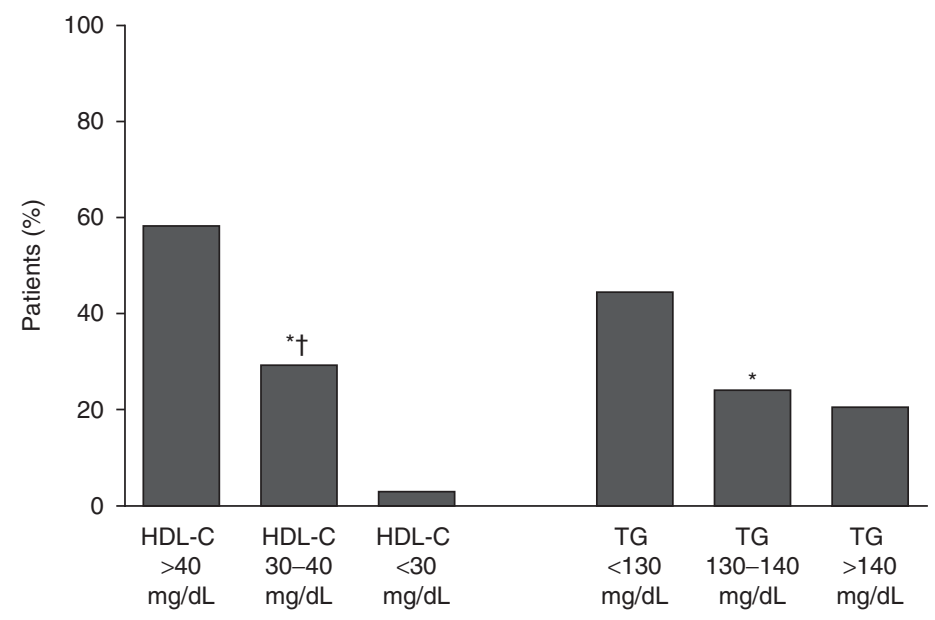

Fig. 4. Prevalence of normal, high-normal ('borderline') and abnormal thresholds for high-density lipoprotein cholesterol (HDL-C) and triglyceride (TG) levels. Data are expressed as percentages of the overall population sample and according to gender distribution. Thresholds for HDL-C were different in men and women, according to the study protocol. ${ }^{*} p<0.05$ vs normal thresholds; $+p<0.05$ vs abnormal thresholds.

explain the lower prevalence of individuals showing borderline levels of BP (19\% in men and 14\% in women) in the Progetto Cuore $^{[21]}$ than those reported in our analysis $(43.5 \%$ in men and $37.4 \%$ in women for the systolic BP, and $34.9 \%$ in men and $25.5 \%$ in women for the diastolic BP levels). The same differences can be observed for those subjects showing abnormal glucose profile; this proportion was lower in the Progetto Cuore $^{[21]}(8 \%$ in men and in $4 \%$ women, taking $110-126 \mathrm{mg} / \mathrm{dL}$ as reference values) than in our analysis $(22.2 \%$ in men and $15.9 \%$ in women, considering $100-110 \mathrm{mg} / \mathrm{dL}$ as reference values). On the other hand, our findings are confident with the results of a recent meta-survey of observational studies performed in Italy, which reported a significantly higher proportion of elevated values of systolic BP with respect to elevation of diastolic BP levels in the setting of general medicine. A similar prevalence of diabetes and hypercholesterolaemia was reported, although this referred to hypertensive patients followed in different clinical settings rather than to outpatients followed by general practitioners as in the present investigation. ${ }^{[22]}$

A second aspect that is of particular relevance is the unexpectedly and relatively high prevalence of individuals with borderline values of major cardiovascular and metabolic risk factors compared with those having normal values of these parameters in a setting of an otherwise healthy general population. In our analysis, in fact, prevalence of individuals having borderline systolic BP levels was comparable with that of subjects showing normal systolic BP levels, but significantly more prevalent than those with abnormal systolic BP levels (i.e. hypertensive patients), with these differences being more evident in men than in women. The same trends were observed for diastolic BP levels, LDL-C, HDL-C and BMI values. These aspects may at least, in part, contribute to explain the relatively higher risk of developing major $\mathrm{CV}$ events, including myocardial infarction, stroke and $\mathrm{CV}$ death, observed in the general population of asymptomatic individuals than that reported in those patients with previous history of (symptomatic) heart or vascular disease. ${ }^{[23]}$ In this view, our results are consistent with several recommendations of current international guidelines, which promote a closer scrutiny for global cardiovascular risk stratification, in order to early identify and promptly treat those (asymptomatic) subjects at potentially high cardiovascular risk before the occurrence of major cardiovascular events. ${ }^{[16,24]}$

A third aspect that is of particular relevance is the distribution of individuals with abnormal lipid profile. While only a minority of the subjects showed abnormal LDL-C levels, about one-third of the population had borderline values of HDL-C and triglycerides. By adding these abnormal parameters to high-normal BP levels (about 35-40\%), impaired glucose regulation (about 20\%) and overweight (about 41\%), and considering the average value of waist circumference (about $97 \mathrm{~cm}$ ), we can reasonably estimate that about $30-35 \%$ of total population sample included in our analysis may have had a metabolic syndrome, according to current diagnostic criteria. ${ }^{[17,18]}$ This condition may significantly increase not only the risk of developing major cardiovascular events, but mostly the 5-10 year risk of new-onset diabetes. ${ }^{[25]}$ Thus, our results suggest closer attention to identifying those subjects having metabolic syndrome, in order to reduce their cardiovascular and metabolic risk.

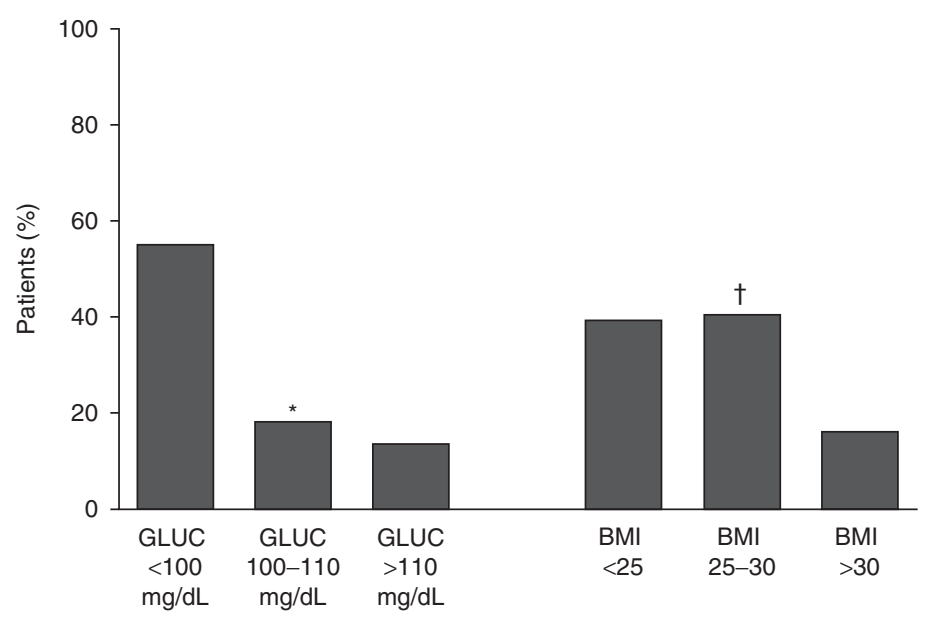

Fig. 5. Prevalence of normal, high-normal ('borderline') and abnormal thresholds for fasting glucose levels (GLUC) and body mass index (BMI). Data are expressed as percentages of the overall population sample. $* p<0.05$ vs normal thresholds; $+p<0.05$ vs abnormal thresholds. 
A final aspect that deserves to be discussed is the relatively high prevalence of subjects with abnormal glucose regulation. This condition, which includes two clinical settings, i.e. fasting hyperglycaemia (IFG) and impaired glucose tolerance (IGT), may be considered as intermediate stages between normal glucose homeostasis and diabetes. Although in our analysis we only considered fasting glucose levels to define subjects with normal, borderline or abnormal glucose profile, several reports indicated that the prevalence of IFG and IGT (or both) accounted for $20 \%$ of the general adult population, ${ }^{[26]}$ which was comparable with that observed in our population (about 20\%). The considerable prevalence of this condition may be of particular interest, because within 3-5 years about $25 \%$ of subjects with abnormal glucose regulation may develop diabetes, $50 \%$ continue to have an abnormal glucose profile, while the remaining $25 \%$ may regress to a normal glucose tolerance. ${ }^{[27]}$

\section{Conclusions}

There is a close, linear relationship between normal, borderline and abnormal values of cardiovascular and metabolic risk factors and increased incidence of cardiovascular diseases. While it is widely accepted that the presence of hypertension, hypercholesterolaemia, obesity and diabetes significantly increases cardiovascular morbidity and mortality, it has been recently demonstrated that even levels below those thresholds traditionally considered for the diagnosis of these clinical conditions, i.e. borderline values, are associated with a significantly higher cardiovascular risk compared with that reported in the normal range of these parameters. In the general population, in fact, a linear relationship between BP, total cholesterol and LDL-C, BMI, glucose levels and cardiovascular complications has been described, and these associations appeared to be even more significant when these abnormalities clustered in the same individuals.

Our results demonstrate a relatively high prevalence of borderline values of major cardiovascular risk factors, which is at least comparable with that of normal thresholds, but higher than that of abnormal values in the setting of general practice in Italy. Since early and effective interventions on major cardiovascular risk factors have demonstrated a favourable influence on cardiovascular disease outcomes, our findings may have a potential impact for future prevention strategies for reducing the overall burden of cardiovascular disease in Italy, by targeting diagnostic and therapeutic interventions to those asymptomatic individuals with borderline abnormalities of cardiovascular risk profile, who are at increased risk of developing major cardiovascular events.

\section{Acknowledgements}

The authors wish to thank all the involved physicians for their contribution to this project (see below). The BORDERLINE study was conducted with an education grant from Science Promotion to the Italian Society of Cardiovascular Prevention. The present study was endorsed by the Italian Society of Cardiovascular Prevention (SIPREC). The authors have no conflicts of interest to disclose.

\section{List of Investigators}

Campania: Rullo Vincenzo, Serrentino Giuseppe. Emilia Romagna: Casadei Massimo, Foresti Alessandra, Linsalata Isabella, Lolli Graziano, Zoccoli Giuseppe. Marche: Antognoni Luca, Bandini Fausto, Cantarini Maurizio, Caruso Michele, Cimarelli Antonella, Giri Mauro, Gregorio Maurizio, Iommi Silvio, Paolinelli Maria Grazia, Pierangeli Stefano, Pietroni Stefania, Pini Annalisa, Vitali Bianca Chiara. Lazio: Agapito Simeoni, Alfieri Vittorio, Bernabè Paolo, Ciula Ottavio, Codipietro Gianluigi, D'Amicis Pietro, De Angelis Vincenzo, Impelluso Rita, Morelli Rossella, Spagnolo Antonio, Virili Mauro. Lombardia: Carotti Gianfranco, Centanni Luigi, Nassuato Marco, Pasquinucci Eugenio, Pozzi Alberto, Trevisan Gianpaolo. Sardegna: Piro Silverio, Putzu Massimo, Ragaglia Michelangelo, Spano Gianni, Spano Salvatore. Veneto: Beccari Alessandro, Carpenè Guido, Danieli Maurizio, Draghi Margherita, Francheo Roberto, Franzoso Federico, Giarrizzo Giuseppe, Pizzulli Nicola, Sandon Dina, Scarin Vladimiro, Tognato Paolo.

\section{References}

1. Kotseva K, Wood D, De Backer G, et al. Cardiovascular prevention guidelines in daily practice: a comparison of EUROASPIRE I, II, and III surveys in eight European countries. Lancet 2009 Mar 14; 373 (9667): 929-40

2. Volpe M. 2008 White paper for implementing strategies and interventions for cardiovascular prevention in Italy. High Blood Press Cardiovasc Prev 2008; 15 (2): $63-73$

3. Morrell J, Zeymer U, Baumgartner I, et al. Differences in management and outcomes between male and female patients with atherothrombotic disease: results from the REACH Registry in Europe. Eur J Cardiovasc Prev Rehabil 2011 Apr; 18 (2): 270-7

4. Volpe M, Erhardt LR, Williams B. Managing cardiovascular risk: the need for change. J Hum Hypertens 2008 Feb; 22 (2): 154-7

5. Gaede $\mathrm{P}$, Lund-Andersen $\mathrm{H}$, Parving $\mathrm{HH}$, et al. Effect of a multifactorial intervention on mortality in type 2 diabetes. N Engl J Med 2008 Feb 7; 358 (6): $580-91$

6. Gaede P, Vedel P, Larsen N, et al. Multifactorial intervention and cardiovascular disease in patients with type 2 diabetes. N Engl J Med 2003 Jan 30; 348 (5): 383-93

7. Dahlof B, Sever PS, Poulter NR, et al. Prevention of cardiovascular events with an antihypertensive regimen of amlodipine adding perindopril as required versus atenolol adding bendroflumethiazide as required, in the Anglo-Scandinavian Cardiac Outcomes Trial-Blood Pressure Lowering Arm (ASCOT-BPLA): a multicentre randomised controlled trial. Lancet 2005 Sep 10-16; 366 (9489): 895-906

8. Sever PS, Dahlof B, Poulter NR, et al. Prevention of coronary and stroke events with atorvastatin in hypertensive patients who have average or lowerthan-average cholesterol concentrations, in the Anglo-Scandinavian Cardiac Outcomes Trial-Lipid Lowering Arm (ASCOT-LLA): a multicentre randomised controlled trial. Lancet 2003 Apr 5; 361 (9364): 1149-58

9. Manuel DG, Lim J, Tanuseputro P, et al. Revisiting Rose: strategies for reducing coronary heart disease. BMJ 2006 Mar 18; 332 (7542): 659-62 
10. Sasamura H, Nakaya H, Julius S, et al. The short treatment with the angiotensin receptor blocker candesartan surveyed by telemedicine (STAR CAST) study: rationale and study design. Hypertens Res 2008 Oct; 31 (10): 1843-9

11. Ren J, Dominguez LJ, Sowers JR, et al. Metformin but not glyburide prevents high glucose-induced abnormalities in relaxation and intracellular $\mathrm{Ca} 2+$ transients in adult rat ventricular myocytes. Diabetes 1999 Oct; 48 (10): 2059-65

12. Landin K, Tengborn L, Smith U. Treating insulin resistance in hypertension with metformin reduces both blood pressure and metabolic risk factors. J Intern Med 1991 Feb; 229 (2): 181-7

13. Sasaki T, Noda Y, Yasuoka Y, et al. Comparison of the effects of telmisartan and olmesartan on home blood pressure, glucose, and lipid profiles in patients with hypertension, chronic heart failure, and metabolic syndrome. Hypertens Res 2008 May; 31 (5): 921-9

14. Ichikawa Y. Comparative effects of telmisartan and valsartan on insulin resistance in hypertensive patients with metabolic syndrome. Intern Med 2007; 46 (17): $1331-6$

15. Bahadir O, Uzunlulu M, Oguz A, et al. Effects of telmisartan and losartan on insulin resistance in hypertensive patients with metabolic syndrome. Hypertens Res 2007 Jan; 30 (1): 49-53

16. Mancia G, De Backer G, Dominiczak A, et al. 2007 ESH-ESC Practice Guidelines for the Management of Arterial Hypertension: ESH-ESC Task Force on the Management of Arterial Hypertension. J Hypertens 2007 Sep; 25 (9): 1751-62

17. Grundy SM, Cleeman JI, Merz CN, et al. Implications of recent clinical trials for the National Cholesterol Education Program Adult Treatment Panel III guidelines. Arterioscler Thromb Vasc Biol 2004 Aug; 24 (8): e149-61

18. Sesti G, Volpe M, Cosentino F, et al. Metabolic syndrome: diagnosis and clinical management: an Official Document of the Working Group of the Italian Society of Cardiovascular Prevention (SIPREC). High Blood Press Cardiovasc Prev 2006; 13 (4): 185-98

19. Ryden L, Standl E, Bartnik M, et al. Guidelines on diabetes, pre-diabetes, and cardiovascular diseases: executive summary. The Task Force on Diabetes and Cardiovascular Diseases of the European Society of Cardiology (ESC) and of the European Association for the Study of Diabetes (EASD). Eur Heart J 2007 Jan; 28 (1): 88-136
20. Volpe M, Borghi C, Cavallo Perin P, et al. Cardiovascular prevention in subjects with impaired fasting glucose or impaired glucose tolerance. High Blood Press Cardiovasc Prev 2010; 17 (2): 73-102

21. Palmieri L, Panico S, Vanuzzo D, et al. Evaluation of the global cardiovascular absolute risk: the Progetto CUORE individual score. Ann Ist Super Sanita 2004; 40 (4): 393-9

22. Volpe M, Tocci G, Trimarco B, et al. Blood pressure control in Italy: results of recent surveys on hypertension. J Hypertens 2007 Jul; 25 (7): 1491-8

23. Lloyd-Jones D, Adams RJ, Brown TM, et al. Heart disease and stroke statistics: 2010 update - a report from the American Heart Association. Circulation 2010 Feb 23; 121 (7): e46-215

24. Graham I, Atar D, Borch-Johnsen K, et al. European guidelines on cardiovascular disease prevention in clinical practice: full text. Fourth Joint Task Force of the European Society of Cardiology and other societies on cardiovascular disease prevention in clinical practice (constituted by representatives of nine societies and by invited experts). Eur J Cardiovasc Prev Rehabil 2007 Sep; 14 Suppl. 2: S1-113

25. Sattar N, Gaw A, Scherbakova O, et al. Metabolic syndrome with and without C-reactive protein as a predictor of coronary heart disease and diabetes in the West of Scotland Coronary Prevention Study. Circulation 2003 Jul 29; 108 (4): 414-9

26. DECODE Study Group on behalf of the European Diabetes Epidemiology Study Group. Will new diagnostic criteria for diabetes mellitus change phenotype of patients with diabetes? Reanalysis of European epidemiological data. BMJ 1998 Aug 8; 317 (7155): 371-5

27. Gerstein HC, Santaguida $P$, Raina $P$, et al. Annual incidence and relative risk of diabetes in people with various categories of dysglycemia: a systematic overview and meta-analysis of prospective studies. Diabetes Res Clin Pract 2007 Dec; 78 (3): 305-12

Correspondence: Professor Massimo Volpe, Division of Cardiology, Department of Clinical and Molecular Medicine, Faculty of Medicine, University of Rome "Sapienza", Sant'Andrea Hospital, Via di Grottarossa, 1035-9, 00189 Rome, Italy.

E-mail: massimo.volpe@uniroma1.it 